\title{
Response to drugs and diet in a compound heterozygote for familial hypercholesterolaemia
}

\author{
V Gudnason, D P R Muller, J K Lloyd, S E Humphries
}

\begin{abstract}
A boy with a total plasma cholesterol concentration of $20.9 \mathrm{mmol} / \mathrm{l}$ which fell significantly with a low fat diet, cholestyramine and simvastatin, was shown to have two different mutations in the low density lipoprotein receptor gene, demonstrating that some patients with homozygous familial hypercholesterolaemia show a good lipid lowering response to treatment.

(Arch Dis Child 1995; 73: 538-540)
\end{abstract}

Keywords: homozygous familial

hypercholesterolaemia, compound heterozygote, LDL receptor mutation, 3-hydroxy-3-methyl-glutaryl CoA reductase inhibitors.

Familial hypercholesterolaemia is a common dominant disorder in most populations, with a heterozygote frequency of approximately one in 500. It is caused by mutations in the low density lipoprotein (LDL) receptor gene; the LDL receptor being responsible for clearance of LDL particles from plasma. ${ }^{1}$ Heterozygotes generally have a two to threefold increase of plasma cholesterol concentrations and premature coronary artery disease. Homozygotes ${ }^{\star}$ are found at a frequency of approximately one in a million and are much more severely affected, with typically a fivefold increased concentration of plasma cholesterol and coronary artery disease at an early age with death from myocardial infarction in the second decade of life. ${ }^{1}$ Patients with homozygous familial hypercholesterolaemia are usually resistant to treatment with diet and drugs. ${ }^{1}$

We report here the clinical and biochemical features of a child who is a compound heterozygote for familial hypercholesterolaemia, and who showed a good response to treatment. The molecular identification of the specific defects causing the disease are also described.

Centre for Genetics of Cardiovascular Disorders,

Department of Medicine, The Rayne Institute, University College of London Medical School, 5 University Street, London WC1E 6JJ

V Gudnason

$S$ E Humphries

Institute of Child

Health, London

D P R Muller

J K Lloyd

Correspondence to: Dr Gudnason.

Accepted 12 July 1995

\section{Case report}

A boy born in 1981 was noted by his mother to have raised lesions behind his knees at the age of 4 years. At the age of 7 he developed a further lesion on his left elbow followed by lesions on his knee. The plasma cholesterol concentration at that time was $20.9 \mathrm{mmol} / 1$. He was placed on a low fat diet and referred to a paediatric lipid clinic. Within one month and on diet alone his cholesterol fell to

^In this paper homozygous familial hypercholesterolaemia will refer to patients with mutations of the LDL receptor gene on refer to patients with mutations of the LDL recepto
both alleles, which may or may not be identical.
$15.9 \mathrm{mmol} / \mathrm{l}$. He was started on cholestyramine ( $8 \mathrm{~g}$ twice/day) and simvastatin (10 $\mathrm{mg} /$ day) and over a three month period his plasma cholesterol concentration fell to $7 \cdot 5$ $\mathrm{mmol} / \mathrm{l}$. Over the next 15 months plasma cholesterol concentrations gradually increased to $12.6 \mathrm{mmol} / 1$ and have remained around this level despite increasing the simvastatin dosage to $30 \mathrm{mg}$ /day (fig 1). On treatment his skin and tendon xanthomata, which were present on the extensor tendons of both middle fingers, had regressed and a coronary angiography done when he was 10 years old showed only minor irregularities in the left coronary artery.

The pedigree of the family is shown in fig 2 . The maternal grandfather had had a myocardial infarction at the age of 54 ; the mother had a total cholesterol concentration of $12 \cdot 0$ $\mathrm{mmol} / \mathrm{l}$ but was symptom free. No information was available on the paternal side.

\section{Molecular analyses}

DNA extraction and Southern blot analysis was carried out as described before. ${ }^{2}$ Amplification by the polymerase chain reaction (PCR) was performed using a described primer set. ${ }^{3}$ Direct sequencing of the amplified DNA was performed as described by Gudnason et $a l,{ }^{4}$ as was the detection of familial defective apolipoprotein B mutation and the apolipoprotein E genotyping. ${ }^{4} \mathrm{LDL}$ receptor protein analysis, was carried out as described by Knight et al. ${ }^{5}$

\section{Results}

Analysis of the patient's DNA by Southern blotting revealed a deletion of exons 15-18 (fig

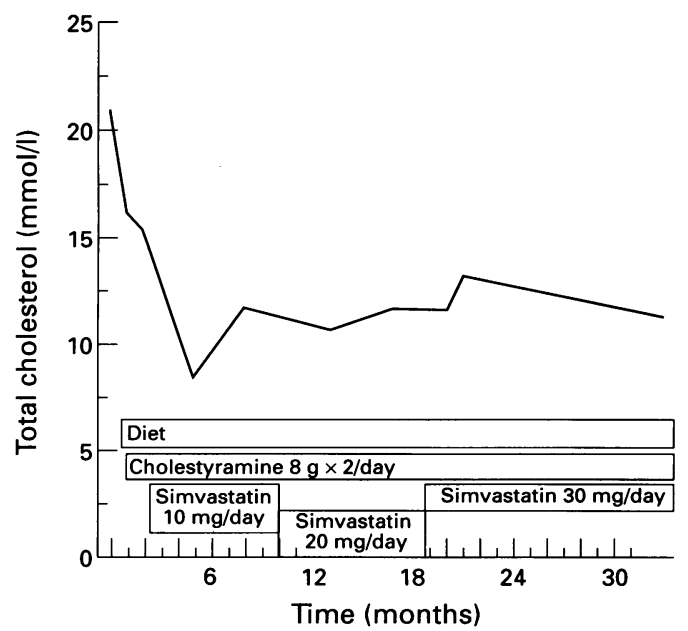

Figure 1 Effect of treatment with diet and drugs on total and LDL plasma cholesterol concentrations. 


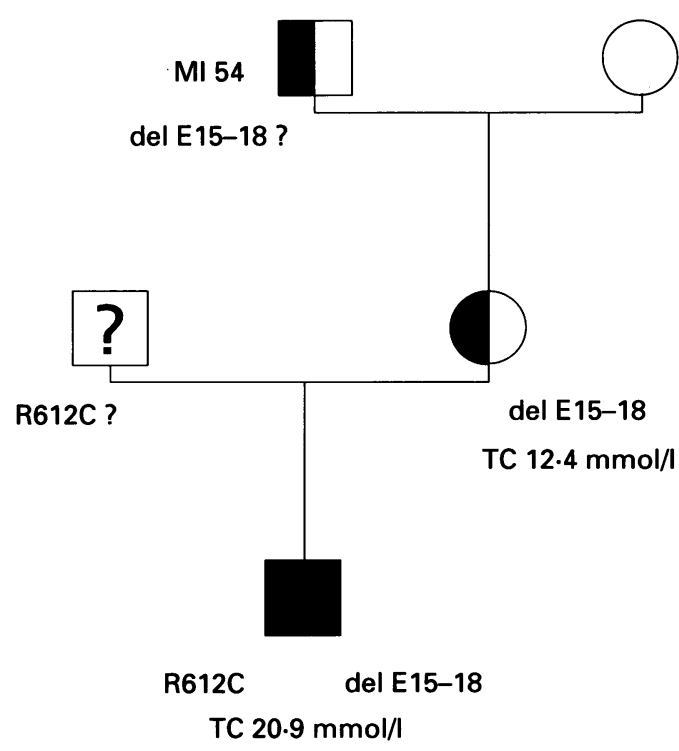

Figure 2 Pedigree of the family; information from the boy's father was not available. (TC=total cholesterol.)

3A) and the same deletion was found in the mother (not shown). The exact details of the deletion are described elsewhere. ${ }^{2}$ The other mutation in the boy was identified by direct sequencing of the amplified fragments after PCR. A novel single base substitution $C$ to $T$ in exon 13 was found, resulting in the substitution of an arginine for cysteine in the epidermal growth factor homology region (fig 3B). Familial defective apolipoprotein $B$ was excluded as a reason for the raised cholesterol concentrations in both the boy and his mother. The apolipoprotein E genotype was E4/3.

An immunoblot of the LDL receptor from lymphoblastoid cells from the boy and his mother (fig 3C) showed an accumulation of a protein of approximately 100 kilodaltons ( $\mathrm{kd}$ ) in both individuals. There was also a normal sized protein in the cells from both the mother and her son, although the amount was reduced in the latter. Further analysis was not possible as no more cells were available.

\section{Discussion}

The good lipid lowering response to dietary advice and drug treatment in the boy raised the question as to whether the patient was homozygous for mutations in the LDL receptor gene or a severely affected heterozygote. In this case the DNA analysis enabled us to show that the patient was a compound heterozygote for familial hypercholesterolaemia.

Immunoblot analysis on lymphoblastoid cells from the boy and his mother, who also carried the large deletion, showed an accumulation of a protein of approximately $100 \mathrm{kd}$ protein. It is likely that this protein accumulated within the cell because the transmembrane portion of the protein was deleted by the truncation but sequences that retain the protein within the cell were not. This mutation therefore resembles the Lebanese mutation which causes a premature stop codon in amino acid 660 in exon 14, and which has been shown to result in the accumulation of a truncated protein in the Golgi compartment, ${ }^{6}$ which has no residual receptor activity. ${ }^{7}$ This indicates that the response to treatment in the boy may be accounted for by the other allele, which is predicted to produce a normal sized protein of approximately $160 \mathrm{kd}$, and could be

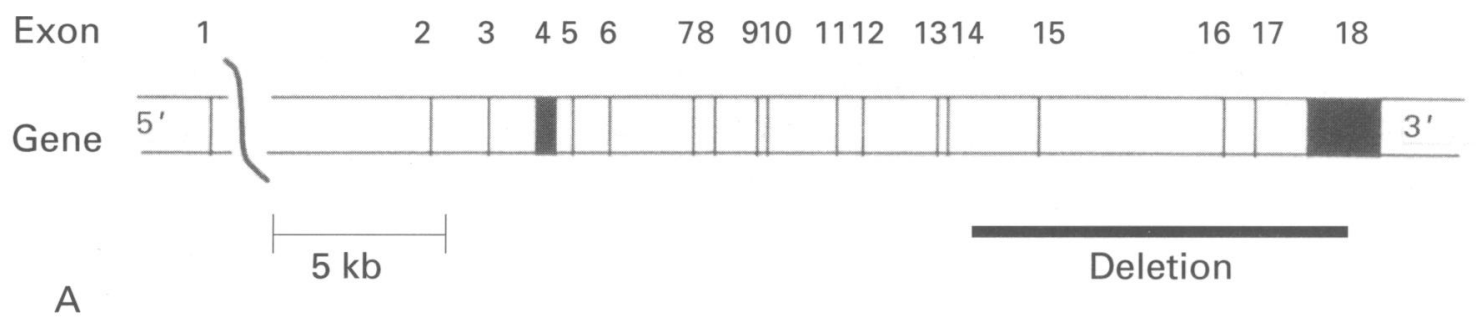

A
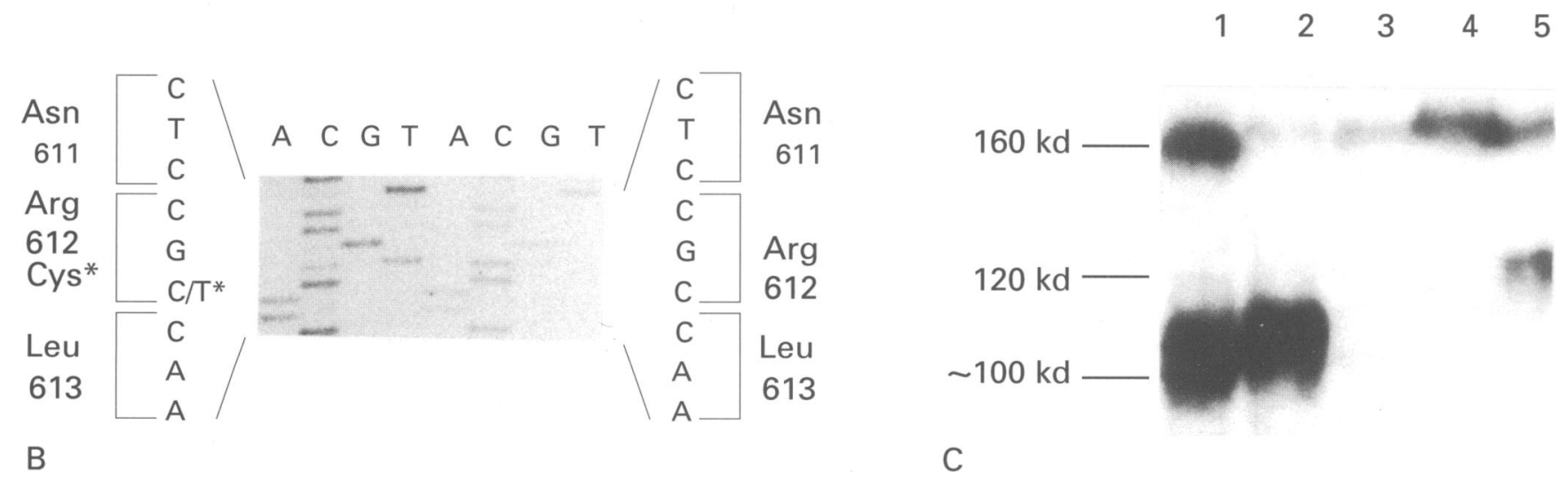

C

Figure $3 D N A$ and protein analysis of the LDL receptor and its gene, in a compound heterozygote for familial hypercholesterolaemia. (A) Map of the $L D L$ receptor gene showing the relative position of the exons and introns. The deletion that spans intron 14 to mid exon 18 is shown as a filled box beneath the map. (B) $A$ sequence of a part of exon 13 in the patient with familial hypercholesterolaemia and his mother showing a $C$ to $T$ transition resulting in $a$ substitution of an arginine for a cysteine in codon 612 is shown. (C) Immunoblotting of extracts from cultured lymphoblastoid cell lines from (1) the mother, (2) the patient, (3) and (4) from normal cells, and (5) from an individual with the P664L mutation showing accumulation of the normally sized precursor protein. 
detected at low levels in the lymphoblastoid cells of the patient (fig $3 \mathrm{C}$ ). As further functional studies were not undertaken it is not known if there was delayed processing of the receptor protein from the precursor to the mature form during passage to the cell surface. The additional cysteine replacing an arginine might have such an effect but there was no evidence for accumulation of a normal sized precursor protein in the cells from the boy. It is also not known whether either of the mutated proteins retained any binding ability. In the light of the good response to treatment seen in this patient it is conceivable that the R612C mutation has some residual activity and a true homozygote for that mutation would respond even better than the compound heterozygote described here. Likewise, it may be predicted that a heterozygote for this mutation will have lower plasma cholesterol concentration as seems to be the case in heterozygous familial hypercholesterolaemia patients with mutations with some residual receptor activity. ${ }^{4}$

There are a growing number of reports of phenotypically homozygous patients who have shown an unexpectedly good response to treatment. ${ }^{1}$ This is usually related to the level of residual LDL receptor activity, ${ }^{8}$ but there is also a recent report of a receptor negative homozygote with familial hypercholesterolaemia who responded well to simvastatin treatment with a fall in plasma cholesterol concentrations of approximately $40 \%$, which was stable over a two year period. ${ }^{9}$ There is, therefore, still much to be learned about the response to treatment of patients with homozygous familial hypercholesterolaemia.

We thank the Department of Chemical Pathology, Salisbury General Infirmary for carrying out the routine lipid analyses. This work was supported by the British Heart Foundation (RG5), the John Pinto Foundation, and the Helen Eppel Fund. We thank Drs B Knight, A K Soutar, and D Patel at the MRC lipoprotein team, Hammersmith Hospital for the labelled monoclonal antibody and expert advice and support with the molecular analysis.

1 Goldstein JL, Brown MS. Familial hyercholesterolaemia. In: Scriver CR, Beaudet AL, Sly WS, Valle D, eds. The metabolic basis of inherited disease. 6th Ed. New York: McGraw Hill, 1989: 1215-50.

2 Sun X-I, Webb JC, Gudnason V, et al. Characterization of deletions in the LDL receptor gene in patients with familial hypercholesterolaemia in the United Kingdom. Arterioscler Thromb 1992; 12: 767-70.

3 Leitersdorf E, Tobin EJ, Davignon J, Hobbs HH. Common low-density lipoprotein receptor mutations in the French Canadian population. 7 Clin Invest 1990; 85: 1014-23.

4 Gudnason V, Day INM, Humphries SE. Effect on plasm lipid levels of mutations in exon 4 of the low density lipoprotein receptor gene in patients with FH. Arterioscler Thromb 1994;14: 1717-22.

5 Knight B, Gavigan SJP, Soutar AK, Patel D. Defective processing and binding of low density lipoprotein receptor in fibroblasts from familial hypercholesterolaemic subject. Eur F Biochem 1989; 179: 639-48.

6 Lehrman MA, Schneider WJ, Brown MS, et al. The Lebanese allele at the low density lipoprotein receptor locus: nonsense mutation produces truncated receptor that is retained in endoplasmic reticulum. Clin Genet 1987; 40: 17-28.

7 Hobbs HH, Brown MS, Goldstein JL. Molecular genetics of the LDL receptor gene in familial hypercholesterolemia. Human Mutation 1992; 1: 445-66.

8 Sprecher DL, Hoeg JM, Schaefer EJ, et al. The association of LDL receptor activity, LCL cholesterol level, and clinical course in homozygous familial hypercholesterolemia. Metabolism 1985; 34: 294-9.

9 Feher MD, Webb JC, Patel DD, et al. Cholesterol-lowering drug therapy in a patient with receptor-negative homozygous familial hypercholesterolaemia. Atherosclerosis 1993; 103: $171-80$. 